\title{
Complications of guided surgery and immediate loading in oral implantology: A report of 12 cases
}

\author{
Marta Abad-Gallegos ${ }^{1}$, Laila Gómez-Santos ${ }^{1}$, M. Angeles Sánchez-Garcés ${ }^{2}$, Manuel Piñera-Penalva ${ }^{3}$, Jordi \\ Freixes-Gil ${ }^{4}$, Ana Castro-García ${ }^{5}$, Cosme Gay-Escoda ${ }^{6}$
}

\begin{abstract}
${ }^{1}$ DDS. Resident of the Master in Oral Surgery and Implantology. University of Barcelona Dental School
${ }^{2} \mathrm{MD}$, DDS, PhD. Master in Oral Surgery and Implantology. Associate Professor of Oral Surgery. Professor of the Master in Oral Surgery and Implantology. University of Barcelona Dental School. Investigator of the IDIBELL Institute

${ }^{3}$ MD, DDS. Master in Oral Surgery and Implantology. Professor of the Master in Oral Surgery and Implantology. University of Barcelona Dental School. Investigator of the IDIBELL Institute

${ }^{4}$ DDS. Professor of the Master in Oral Surgery and Implantology. University of Barcelona Dental School. Investigator of the IDIBELL Institute

${ }^{5}$ MD, DDS. Professor of the Master in Oral Surgery and Implantology. University of Barcelona Dental School. Investigator of the IDIBELL Institute

${ }^{6} \mathrm{MD}$, DDS, PhD. Chairman of Oral and Maxillofacial Surgery. Director and professor of the Master in Oral Surgery and Implantology. University of Barcelona Dental School. Coordinating investigator of the IDIBELL Institute. Co-director of the TMJ and Orofacial Pain Unit. Head of the Service of Maxillofacial Surgery, Teknon Medical Center. Barcelona, Spain
\end{abstract}

Correspondence:

Centro Médico Teknon

Instituto de Investigación IDIBELL

C/Vilana 12

08022 - Barcelona (Spain)

cgay@ub.es

Received: 28/02/2010

Accepted: 30/06/2010
Abad-Gallegos M, Gómez-Santos L, Sánchez-Garcés MA, Piñera-Penalva M, Freixes-Gil J, Castro-García A, Gay-Escoda C. Complications of guided surgery and immediate loading in oral implantology: A report of 12 cases. Med Oral Patol Oral Cir Bucal. 2011 Mar 1;16 (2):e220-4. http://www.medicinaoral.com/medoralfree01/v16i2/medoralv16i2p220.pdf

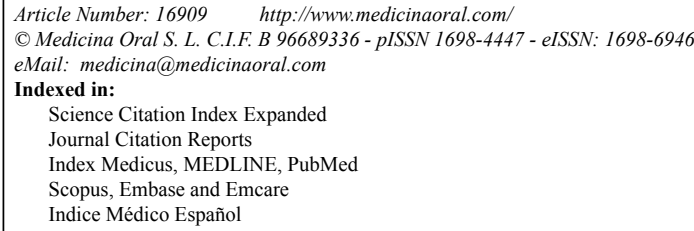

\begin{abstract}
Objectives: The growing interest in minimally invasive surgery, together with the possibility of fitting prostheses with immediate function, have led to the development of software capable of planning and manufacturing a surgical guide and prosthesis that can be placed upon conclusion of the implant surgery step.

The present study evaluates the surgical and prosthetic complications of implant treatment with the guided surgery technique, together with patient comfort during and after treatment.

Patients and methods: A retrospective observational study was made of 19 patients with partially or totally edentulous upper and/or lower maxillae, involving the placement of a total of 122 implants. All cases were planned and operated upon with the guided surgery technique.

Results: A total of 122 implants were placed in 14 males and 5 females. The intraoperative surgical complications comprised a lack of primary stability, while the postoperative complications consisted of infections and a lack of implant osteointegration. Ten implants failed. The prosthetic complications in turn comprised loosening of the provisional prosthesis screws, prosthesis tooth fracture, and a lack of passive fit of the immediate prosthesis. The degree of patient satisfaction was evaluated using a verbal scale.

Conclusions: Implant restoration with the guided surgery technique and immediate functional loading is a predictable procedure, provided patient selection and the surgical technique are adequate, affording lesser postoperative morbidity and increased patient satisfaction thanks to the immediate restoration of esthetics and function.
\end{abstract}

Key words: Guided surgery, immediate loading, dental implants, complications. 


\section{Introduction}

The growing interest in flapless implant surgery, together with the possibility of fitting prostheses with immediate function in edentulous patients, have led to the development of software capable of planning and manufacturing a surgical guide and prosthesis that can be placed immediately after implant insertion (1).

Following flap raising, bone reabsorption takes place, and the resulting reduction in crest height is related to the bone width of the zone involved (2). The flapless technique maintains the periosteum and blood supply to the bone, avoids modification of the gingival profile following the contour of the surgical incision, and shortens the surgery time $(3,4)$. This technique usually increases the success of immediate loading due to preservation of the blood supply, and reduces postoperative discomfort for the patients (3). Careful implant placement in the surgical bed, taking into account both the patient anatomy and the type of definitive prosthesis involved, is a key factor for treatment success $(5,6)$. The surgical guide marks the appropriate direction of implant insertion, and can be used not only in situations characterized by anatomical limitations but also under favorable conditions with a view to avoiding possible manual error - thus meeting the requirements for precise prosthetic restoration (6). With this methodology it is possible to avoid inadequate implant positioning - the latter together with mechanical overload being the factors that increase the risk of implant failure and of defects in the esthetic and functional outcome of the restoration (7).

The radiological diagnostic study allows us to evaluate the anatomical characteristics of the upper and lower maxillae. Transfer of the data obtained from imaging explorations such as computed tomography (CT) to software such as Nobel Guide ${ }^{\circledR}$ (Nobel Biocare, Göteborg, Sweden), Simplant ${ }^{\circledR}$ (Materialise Dental, Madrid, Spain), Facilitate ${ }^{\circledR}$ (Astra Tech, S.A, Hospitalet de Llobregat, Barcelona, Spain) or Neoguide ${ }^{\circledR}$ (Nobel Biocare, Göteborg, Sweden) is improving the three-dimensional (3D) planning of treatment, thereby ensuring controlled implant insertion through assisted or guided surgery $(8,9)$.

In this type of surgery, both the safety and success of treatment depend on accuracy.

In implantology, the documentation on this particular variable is still limited, however. In this context, the literature describes coronal or apical deviations of somewhat under $1 \mathrm{~mm}$, with scant variability $(10,11)$. Other authors such as van Steenberghe et al. (12) have shown that the deviations may reach up to $2.7 \mathrm{~mm}$ - deviation of the planned angle with respect to the angle obtained after implant placement being less than three degrees.

The present study evaluates the surgical and prosthetic complications of implant treatment with the computer guided surgery technique (Nobel Guide ${ }^{\circledR}$ ), together with patient comfort during and after treatment.

\section{Patients and Methods}

A retrospective observational study was made of 19 patients (14 males and 5 females, aged 45-79 years) with partially or totally edentulous upper and/or lower maxillae, involving the placement of a total of 122 implants (Table 1).

Table 1. Number and location of the implants in each case and failed implants.

\begin{tabular}{|c|c|c|c|c|}
\cline { 2 - 5 } \multicolumn{1}{c|}{} & \multicolumn{2}{c|}{ Implant number and location } & \multicolumn{2}{c|}{ Failed implants } \\
\cline { 2 - 5 } CASE & Upper maxilla & Mandible & 0 & 0 \\
\hline 1 & 6 & 0 & 0 & 3 \\
\hline 2 & 0 & 6 & 0 & 0 \\
\hline 3 & 4 & 0 & 0 & 0 \\
\hline 4 & 4 & 0 & 0 & 0 \\
\hline 5 & 6 & 0 & 1 & 0 \\
\hline 6 & 4 & 4 & 0 & 0 \\
\hline 7 & 6 & 0 & 0 & 0 \\
\hline 8 & 6 & 0 & 0 & 0 \\
\hline 9 & 0 & 6 & 0 & 0 \\
\hline 10 & 6 & 0 & 0 & 0 \\
\hline 11 & 6 & 6 & 2 & 0 \\
\hline 12 & 6 & 0 & 0 & 0 \\
\hline 13 & 6 & 0 & 0 & 0 \\
\hline 14 & 4 & 0 & 0 & 3 \\
\hline 15 & 6 & 0 & 0 & 0 \\
\hline 16 & 6 & 0 & 0 & 0 \\
\hline 17 & 3 & 0 & 0 & 0 \\
\hline 18 & 5 & 0 & 0 & 0 \\
\hline 19 & & 0 & 0 & 0 \\
\hline
\end{tabular}


Minimally invasive surgery was used in all cases, employing a surgical guide obtained after surgical planning with the software package (Nobel Guide $\AA$ ). This program allows the planning of implant placement thanks to three-dimensional reconstruction of the CT images. The study variables were patient age, gender, number of implants, their location, the type of provisional and definitive prostheses involved, the time wearing the provision prosthesis, surgical and prosthetic complications, and the number of failed implants and their location (Fig. 1).

We also registered patient comfort using a verbal scale ( $0=$ poor, $1=$ regular, $2=$ good, $3=$ very good, $4=$ excellent $)$. The patients were questioned about the immediate postoperative course and the comfort of the provisional and definitive prostheses.

The data obtained were subjected to descriptive analysis using the SPSS version 15.0 statistical package (license of the University of Barcelona, Spain).

\section{Results}

A total of 122 implants were placed in 14 males and 5 females with a mean age of 61.9 years. A total of 92 implants (75.4\%) were placed in the upper maxilla and $30(24.6 \%)$ in the mandible (Table 1).

Ten of the 122 implants failed (8.2\%), corresponding to 5 patients. Four upper maxillary implants failed (3.2\%) in three patients, versus 6 in the mandible (4.9\%)( 3 in one patient and 3 in another)(Table 1). All failures occurred in totally edentulous subjects and after immediate loading. In $70 \%$ of the cases $(n=7)$, failure was due to a lack of osteointegration, while infection was the cause in the remaining $30 \%(\mathrm{n}=3)$. In these cases the implants were removed, followed by replacement using a conventional flap technique 2-4 months later. The implants that failed because of infection had been inserted in locations different from those initially planned.

The provisional prosthesis was a resin bridge in two cases $(13.3 \%)$ and a hybrid prosthesis in 14 (73.3\%). In one of the cases a complete lower prosthesis was placed as provisional measure, while no provisional prosthesis was placed in the remaining two patients. Delayed loading was decided in 5 cases. The mean time wearing the provisional prosthesis was 7.5 months (range 4-13 months).

In the cases of implant failure, the definitive prosthesis was not placed until osteointegration was considered complete (after 3-8 months), and during this period the patients continued to wear the planned prosthesis, but without loading of the implants repositioned in the zones where failure had occurred. In case number 2 , all three implants were replaced after a waiting period of 3-4 months, and classical prosthetic restoration was applied in wait of definitive passive osteointegration (between 3-4 months).

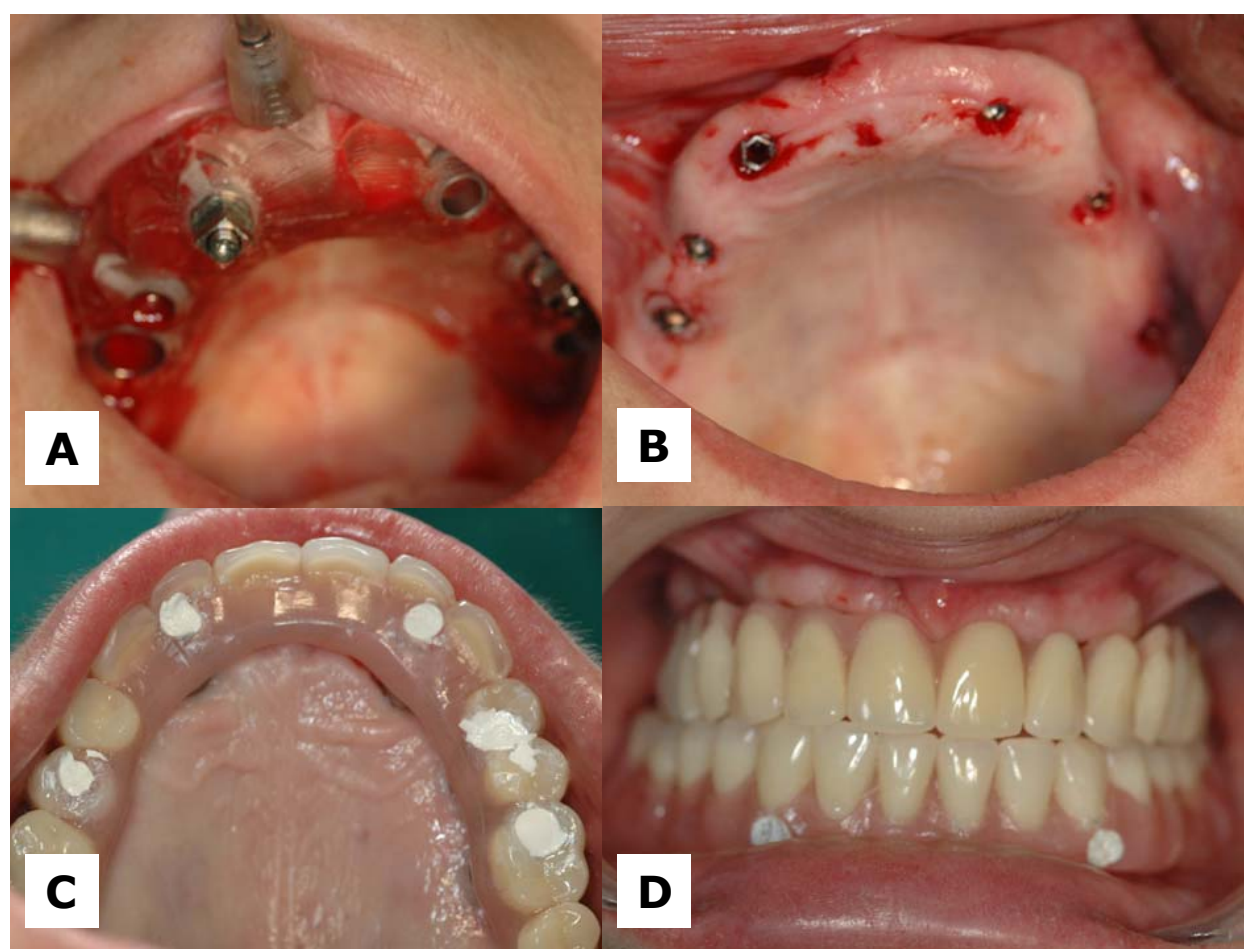

Fig. 1. Guided surgery with immediate loading. (A) Minimally invasive surgery using a software-generated surgical guide. (B) Postoperative view after implant placement in the upper maxilla. (C) View of the upper prosthesis immediate. (D) Final view after surgery and fitting of the upper prosthesis with immediate loading. 
The intraoperative surgical complications comprised a lack of primary stability in 5 cases $(26.3 \%)$, thus precluding programmed immediate loading, and technical difficulties caused by limited oral aperture in one patient. The postoperative complications in turn consisted of a lack of implant osteointegration that led to the failure of 7 implants in four subjects. In one case an implant was lost due to infection, and on raising the flap and examining the adjacent implants, important bone dehiscence caused us to remove two more. All the implants were posteriorly successfully replaced by open surgery. Ten cases $(52.6 \%)$ showed no prosthetic complications. The most frequent such complications were screw loosening of the provisional prosthesis ( 2 cases, $10.5 \%$ )(in only one of them was a cemented prosthesis designed), fracture of the prosthesis or of some of the prosthetic teeth ( 2 cases, $10.5 \%$ ), a lack of passive fit of the immediate prosthesis ( 1 case, $5.3 \%$ ), and implant pain ( 1 case, $5.3 \%$ ). Lastly, in one case implant angulation was not adequate and an angled abutment was positioned.

In four cases $(21.1 \%)$ a lack of primary stability precluded the placement of an immediate provisional prosthesis.

Patient comfort during the immediate postoperative period was described as poor in one case $(5.3 \%)$, good in $8(42.1 \%)$, very good in $6(31.6 \%)$, and excellent in four $(21.1 \%)$. In turn, patient satisfaction with the immediate prosthesis was described as poor in one case (5.3\%), regular in two $(10.5 \%)$, good in $5(26.3 \%)$, very good in $9(47.4 \%)$, and excellent in two (10.5\%). Lastly, patient satisfaction with the definitive prosthesis was described good in 6 cases $(31.6 \%)$, very good in $9(47.4 \%)$, and excellent in four (21.1\%).

\section{Discussion}

Computed radiological imaging guided surgery in implantology aims to plan the operation and its execution with the greatest precision possible (3). Thanks to the three-dimensional diagnostic study it is possible to perform minimally invasive surgery without the need for conventional supracrestal incision or the raising of a mucoperiosteal flap - thereby favoring good sealing of the peri-implant tissues from the start $(4,13)$. Cannizzaro et al. (14) reported a $91.9 \%$ success rate in 33 patients with edentulous upper maxillae subjected to flapless surgery and involving immediate loading protocols. In our series the true success rate was $69.5 \%$, since in $31.5 \%$ of the cases immediate loading was not carried out due to a lack of primary stability, the existence of parafunctional habits, or (in one patient) because the objective from the start was to perform guided surgery with delayed loading. It should be taken into account that despite the benefits of this technique, it cannot be used when immediate post-extraction implant placement is planned; soft tissue redistribution is not possible; and leveling of the alveolar crest to create a platform for positioning the head of the implant cannot be done. In many cases, all these factors are essential for future peri-implant health and for the ultimate esthetic outcome.

According to some authors, the accuracy of computer guided implant surgery can be comparable to that of standard treatment, since a deviation of $1 \mathrm{~mm}$ is irrelevant when considering the recommendation to maintain a minimum safety margin of $2 \mathrm{~mm}$ between the implant and noble structures (10). However, other studies have found that the deviation may reach $2.7 \mathrm{~mm}$ and thus affect the safety margin in some cases (12).

It moreover should be taken into account that this technique may present an accumulation of small errors at different points during the planning process and in application of the surgical technique. As an example, error may occur during CT scanning (since structural differences of under $1 \mathrm{~mm}$ are often not recognized), in manufacturing the surgical guide, and in stabilizing the guide. Accordingly, any deviation results in implant placement at other levels of emergence, and axis deviation may also occur due to the length of the drill. In some cases, the guide (with a thickness of $10 \mathrm{~mm}$ ), does not allow us to place some implants in the posterior zones due to limited oral aperture of the patient $(6,10,12,15)$.

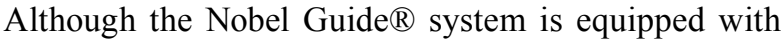
provisional abutments that can be inserted between the prosthesis and the implants to compensate minor vertical discrepancies and modifications in angulation, it cannot compensate horizontal deviations - thereby making immediate placement of the prosthesis impossible, unless it is segmented $(16,17)$. We did not observe this complication in our series. In contrast, we recorded other problems such as a lack of primary stability, marginal bone loss and a lack of osteointegration that likewise did not allow immediate placement of the prosthesis as originally planned. Other complications were screw loss or loosening, and fracture of the prosthesis or of some of its elements. These problems may be related to defective occlusal fitting and/or the existence of parafunctional habits $(16,18)$. The most common complication in our study was loosening of the provisional prosthesis, followed by prosthesis tooth fracture, and a lack of passive fit. There were also cases of bone reabsorption that exceeded the limits for success, and a lack of implant osteointegration. According to Misch et al. (19), such problems may be due to a lack of rigidity of the prosthetic structure, which lacks a metallic base. This would increase the risk of prosthesis fractures, which in turn may adversely affect primary stability of the implants. On the other hand, although the definitive prosthesis could be manufactured with these systems, some authors such as Bedrossian (1) recommend placing a provisional prosthesis first, since this allows patients to inform of their functional and esthetic needs before 
the definitive prosthesis is prepared. We agree with this opinion, for in addition to allowing the final prosthesis to be adjusted to the patient needs, satisfaction with the result of treatment is improved - since most of the prosthetic complications occur during the period in which the patient wears the provisional restoration. This would also favor the cost-efficacy ratio of treatment, since the clinician is able to detect and solve the possible complications before manufacturing the definitive metal base prosthesis. Implant restoration with the guided surgery technique and immediate functional loading is therefore a predictable procedure, provided patient selection and the surgical technique are adequate. A thorough preoperative workup is essential for planning the number and type of implants, as well as the optimum location of implant placement, considering the anatomy of the zone targeted for restoration and the existing bone quality. Prior planning of the definitive prosthesis design is required, with posterior manufacture based on the threedimensional imaging data, and which will facilitate the preparation of a guide for the surgical intervention.

This technique results in lesser postoperative morbidity, since surgery is minimally invasive, and increased patient satisfaction is ensured thanks to the immediate restoration of esthetics and function.

\section{References References with links to Crossref-DOI}

1. Bedrossian E. Laboratory and prosthetic considerations in computer-guided surgery and immediate loading. J Oral Maxillofac Surg. 2007;65:47-52.

2. Campelo LD, Camara JR. Flapless implant surgery: a 10-year clinical retrospective analysis. Int $\mathrm{J}$ Oral Maxillofac Implants. 2002;17:271-6.

3. Fortin T, Bosson JL, Coudert JL, Isidori M. Reliability of preoperative planning of an image-guided system for oral implant placement based on 3-dimensional images: an in vivo study. Int J Oral Maxillofac Implants. 2003;18:886-93.

4. Malo P, de Araujo Nobre M, Lopes A. The use of computer-guided flapless implant surgery and four implants placed in immediate function to support a fixed denture: preliminary results after a mean follow-up period of thirteen months. J Prosthet Dent. 2007;97:S2634.

5. Fortin T, Champleboux G, Lormée J, Coudert JL. Precise dental implant placement in bone using surgical guides in conjunction with medical imaging techniques. J Oral Implantol. 2000;26:300-3.

6. Tahmaseb A, De Clerck R, Wismeijer D. Computer-guided implant placement: 3D planning software, fixed intraoral reference points, and CAD/CAM technology. A case report. Int J Oral Maxillofac Implants. 2009;24:541-6.

7. Ruppin J, Popovic A, Strauss M, Spüntrup E, Steiner A, Stoll C. Evaluation of the accuracy of three different computer-aided surgery systems in dental implantology: optical tracking vs. stereolithographic splint systems. Clin Oral Implants Res. 2008;19:709-16.

8. Brief J, Edinger D, Hassfeld S, Eggers G. Accuracy of image-guided implantology. Clin Oral Implants Res. 2005;16:495-501.

9. van Steenberghe D, Glauser R, Blombäck U, Andersson M, Schutyser F, Pettersson A, et al. A computed tomographic scan-derived customized surgical template and fixed prosthesis for flapless surgery and immediate loading of implants in fully edentulous maxillae: a prospective multicenter study. Clin Implant Dent Relat Res. 2005;7 Suppl 1:S111-20.

10. Valente F, Schiroli G, Sbrenna A. Accuracy of computer-aided oral implant surgery: a clinical and radiographic study. Int J Oral Maxillofac Implants. 2009;24:234-42.

11. Sarment DP, Sukovic P, Clinthorne N. Accuracy of implant placement with a stereolithographic surgical guide. Int J Oral Maxillofac Implants. 2003;18:571-7.

12. Van Steenberghe D, Malevez C, Van Cleynenbreugel J, Bou Serhal C, Dhoore E, Schutyser F, et al. Accuracy of drilling guides for transfer from three-dimensional CT-based planning to placement of zygoma implants in human cadavers. Clin Oral Implants Res. 2003;14:131-6.

13. Sanna AM, Molly L, van Steenberghe D. Immediately loaded CAD-CAM manufactured fixed complete dentures using flapless implant placement procedures: a cohort study of consecutive patients. J Prosthet Dent. 2007;97:331-9.

14. Cannizzaro G, Leone M, Esposito M. Immediate functional loading of implants placed with flapless surgery in the edentulous maxilla: 1-year follow-up of a single cohort study. Int J Oral Maxillofac Implants. 2007;22:87-95.

15. Terzioğlu H, Akkaya M, Ozan O. The use of a computerized tomography-based software program with a flapless surgical technique in implant dentistry: a case report. Int J Oral Maxillofac Implants. 2009;24:137-42.

16. Oyama K, Kan JY, Kleinman AS, Runcharassaeng K, Lozada JL, Goodacre CJ. Misfit of implant fixed complete denture following computer-guided surgery. Int J Oral Maxillofac Implants. 2009;24:124-30.

17. Tan KB, Rubenstein JE, Nicholls JI, Yuodelis RA. Three-dimensional analysis of the casting accuracy of one-piece, osseointegrated implant-retained prostheses. Int J Prosthodont. 1993;6:346-63.

18. Naert I, Quirynen M, van Steenberghe D, Darius P. A study of 589 consecutive implants supporting complete fixed prostheses. Part II: Prosthetic aspects. J Prosthet Dent. 1992;68:949-56.

19. Misch CE, Wang HL, Misch CM, Sharawy M, Lemons J, Judy KW. Rationale for the application of immediate load in implant dentistry: Part I. Implant Dent. 2004;13:207-17.

\section{Acknowledgments}

This study has been carried out by the consolidated research group in "Dental and Maxillofacial Pathology and Treatment" of the Institut d'Investigació Biomèdica de Bellvitge (IDIBELL), with financial support from the oral surgery teaching-healthcare agreement among the University of Barcelona, the Consorci Sanitari Integral and the Servei Català de la Salut of the Generalitat de Catalunya. 